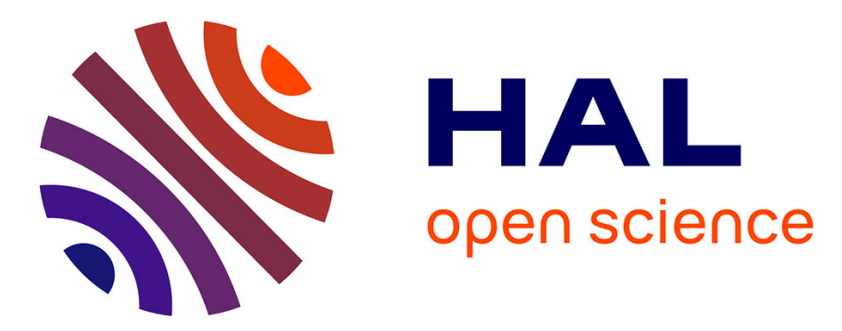

\title{
Continuous-time lossless systems, boundary interpolation and pivot structures
}

Ralf L.M. Peeters, Martine Olivi, Bernard Hanzon

\section{To cite this version:}

Ralf L.M. Peeters, Martine Olivi, Bernard Hanzon. Continuous-time lossless systems, boundary interpolation and pivot structures. SSSC- 5th Symposium on System Structure and Control - 2013, Feb 2013, Grenoble, France. hal-00788428

\section{HAL Id: hal-00788428 \\ https://hal.inria.fr/hal-00788428}

Submitted on 28 Mar 2013

HAL is a multi-disciplinary open access archive for the deposit and dissemination of scientific research documents, whether they are published or not. The documents may come from teaching and research institutions in France or abroad, or from public or private research centers.
L'archive ouverte pluridisciplinaire HAL, est destinée au dépôt et à la diffusion de documents scientifiques de niveau recherche, publiés ou non, émanant des établissements d'enseignement et de recherche français ou étrangers, des laboratoires publics ou privés. 


\title{
Continuous-time lossless systems, boundary interpolation and pivot structures
}

\author{
Ralf L.M. Peeters * Martine Olivi** Bernard Hanzon*** \\ * Dept. Knowl. Engin., Maastricht University, The Netherlands \\ ** INRIA, Proj. APICS, BP 93, 06902 Sophia-Antipolis Cedex, France \\ *** School of Math. Sciences, University College Cork, Cork, Ireland
}

\begin{abstract}
Balanced realizations of lossless systems can be generated from the tangential Schur algorithm using linear fractional transformations. In discrete-time, canonical forms with pivot structures are obtained by interpolation at infinity. In continuous-time case interpolation at infinity is on the stability boundary, leading to an angular derivative interpolation problem. In the scalar case, the balanced canonical form of Ober can be recovered in this way. Here we generalize to the multivariable case. It is shown that boundary interpolation can be regarded as a limit of classical interpolation with interpolation points tending to the imaginary axis. Some pivot structures can be generated, but no complete atlas is obtained. However, for input normal pairs an atlas of admissible pivot structures can be generated in a closely related way.
\end{abstract}

Keywords: Lossless systems, balanced realization, interpolation theory, canonical form, pivot structure, Schur algorithm, linear fractional transformations

\section{INTRODUCTION}

Lossless systems have several applications in linear systems and control theory. In system identification (Bruls et al. (1999)), in $H_{2}$-model order reduction (Leblond and Olivi (1998); Fulcheri and Olivi (1998)), and in digital signal processing (Vaidyanathan and Doğanata (1989); Strang and Nguyen (1996)). They are also central in the construction of parameterizations of various classes of linear systems, see Ober (1987b); Hanzon and Ober (1998); Alpay et al. (1994). In that context balanced realizations of lossless systems have been pursued. See Peeters et al. (2009) for a survey on this, mainly in discrete-time, where the tangential Schur algorithm and related results from interpolation theory (Ball et al. (1990)) are employed.

In Hanzon and Peeters (2000) balanced realizations $(A, B, C, D)$ for the discrete-time scalar case were constructed with a triangular structure in the matrix $[B, A]$ and also in the controllability matrix. Then in Hanzon et al. (2006) the discrete-time multivariable case was presented within the framework of interpolation theory, with interpolation points away from the stability boundary. In Peeters et al. (2007) pivot structures in $[B, A]$ as well as in the controllability matrix were obtained, for interpolation at infinity and with direction vectors subject to certain admissibility conditions. If the admissibility conditions are dropped, then subdiagonal pivot structures in $[B, A]$ arise, but pivot structures in the controllability matrix may be lost, see Hanzon et al. (2009). In Peeters et al. (2010) the discrete-time multivariable case is studied with interpolation at the stability boundary. This construction can be viewed as a limit of classical interpolation.

To obtain parameterizations for lossless systems in continuous-time, the bilinear transformation can be used, but it typically destroys the pivot structures. This is particularly inconvenient for balance-and-truncate type approximation methods, and also for numerical procedures to put a system in canonical form and extract the parameters. A balanced canonical form for continuous-time scalar lossless systems was first presented in Ober (1987a,b). This canonical form has an upper triangular $[B, A]$ and an upper triangular controllability matrix; the matrix $A$ is a Schwarz matrix. In Hanzon et al. (2008) it was shown how it relates to a Schur algorithm with interpolation points on the stability boundary at infinity.

We now complete the picture by addressing the continuoustime multivariable case. In Section 2 we give a background on continuous-time $(J$-)lossless matrices and $(J$-) balanced realizations. In Section 3 we discuss a tangential Schur algorithm in continuous-time, with interpolation points not on the imaginary axis, and associated balanced realizations. In Section 4 the case with interpolation points on the imaginary axis is addressed. In Section 5 Ober's scalar balanced canonical form is discussed and in Section 6 its construction is generalized to the multivariable case. It is found that pivot structures can only be generated for input normal pairs, taking interpolation points at infinity and with direction vectors chosen from the standard basis vectors subject to admissibility conditions. Section 7 concludes the paper. Proofs are largely omitted because of space limitations.

\section{CONTINUOUS-TIME LOSSLESS MATRICES AND BALANCED REALIZATIONS}

We denote by $\Pi^{+}:=\{s \in \mathbb{C} ; \operatorname{Re} s>0\}$ and $\Pi^{-}:=$ $\{s \in \mathbb{C} ; \operatorname{Re} s<0\}$ the open right and left half-planes, respectively. For any matrix function $R(s)$, we define the matrix functions $R^{*}(s)$ and $R^{\sharp}(s)$ by

$$
R^{*}(s):=R(\bar{s})^{*}, \quad \text { and } \quad R^{\sharp}(s):=R^{*}(-s) .
$$


(Here ${ }^{*}$ denotes Hermitian transposition and $\bar{s}$ denotes the complex conjugate of $s$.) Note that if $s$ lies on the imaginary axis, then $R^{\sharp}(s)=R(s)^{*}$.

A $p \times p$ rational function $G(s)$ is called (continuous-time) lossless if at all points of analyticity $s \in \mathbb{C}$ it holds that

$$
\begin{array}{ll}
G(s) G(s)^{*} \leq I_{p} & \text { for all } s \in \Pi^{+}, \\
G(s) G(s)^{*}=I_{p} & \text { for all } s \in i \mathbb{R}, \\
G(s) G(s)^{*} \geq I_{p} & \text { for all } s \in \Pi^{-} .
\end{array}
$$

The function $G(s)$ is lossless if and only if $G^{*}(s)$ is lossless. If $G(s)$ is lossless of (generalized) McMillan degree $n$, then its $n$ poles (including multiplicities) are all in $\Pi^{-}$. The inverse of $G(s)$ exists and is given by $G(s)^{-1}=G^{\sharp}(s)$.

If $(A, B, C, D)$ is a balanced realization of such a lossless function $G(s)$, then it is minimal (i.e., controllable and observable) and it holds that

$$
\begin{aligned}
& D D^{*}=I_{p} \quad(D \text { is } p \times p \text { unitary }), \\
& C=-D B^{*} \quad(\text { of size } p \times n), \\
& A+A^{*}=-B B^{*},
\end{aligned}
$$

so that both Gramians are equal to $I_{n}$. Conversely, if $(A, B, C, D)$ satisfies these three conditions, then the associated transfer function $G(s)=D+C\left(s I_{n}-A\right)^{-1} B$ is lossless of McMillan degree $\leq n$. In that case, the McMillan degree of $G(s)$ equals $n$ if and only if $A$ is (continuous-time) asymptotically stable (i.e., all its eigenvalues are in $\Pi^{-}$), in which case $(A, B, C, D)$ is minimal and balanced.

Note that every lossless $G(s)$ of McMillan degree $n$ admits such a balanced realization $(A, B, C, D)$. Then $(\widetilde{A}, \widetilde{B}, \widetilde{C}, \widetilde{D})$ is another balanced realization of $G(s)$ if and only if $\widetilde{D}=D$ and there exists a (unique) $n \times n$ unitary matrix $Q$ for which $\widetilde{A}=Q A Q^{*}, \widetilde{B}=Q B$ and $\widetilde{C}=C Q^{*}$. Thus, starting from a given (minimal) balanced realization, the set of all (minimal) balanced realizations of $G(s)$ is generated by the set of linear state-space isometries, i.e., by state-space basis transformations from the unitary group $U(n)$.

\subsection{The Grasse product}

Let $(A, B, C, D)$ be a state-space realization of a transfer function $G(s)$, then the associated block-partitioned matrix

$$
R=\left[\begin{array}{ll}
D & C \\
B & A
\end{array}\right]
$$

is called the realization matrix. For two $(p+n) \times(p+n)$ realization matrices $R_{1}=\left[\begin{array}{ll}D_{1} & C_{1} \\ B_{1} & A_{1}\end{array}\right]$ and $R_{2}=\left[\begin{array}{cc}D_{2} & C_{2} \\ B_{2} & A_{2}\end{array}\right]$ (not necessarily minimal and not necessarily corresponding to lossless transfer functions), we define their Grasse product by:

$$
R_{1} \odot R_{2}=\left[\begin{array}{cc}
D_{1} D_{2} & D_{1} C_{2}+C_{1} \\
B_{1} D_{2}+B_{2} & B_{1} C_{2}+A_{1}+A_{2}
\end{array}\right] .
$$

Note that the Grasse product can equivalently be defined in terms of conventional matrix multiplication as:

$$
R_{1} \odot R_{2}=R_{1}\left[\begin{array}{cc}
I_{p} & 0 \\
0 & 0
\end{array}\right] R_{2}+R_{1}\left[\begin{array}{cc}
0 & 0 \\
0 & I_{n}
\end{array}\right]+\left[\begin{array}{cc}
0 & 0 \\
0 & I_{n}
\end{array}\right] R_{2}
$$

Proposition 1. The Grasse product has the following properties:

(a) $\left(R_{1} \odot R_{2}\right) \odot R_{3}=R_{1} \odot\left(R_{2} \odot R_{3}\right)$ (associativity).

(b) The matrix $E=\left[\begin{array}{cc}I_{p} & 0 \\ 0 & 0\end{array}\right]$ is the (unique) neutral element: for all $R$ it holds that $E \odot R=R \odot E=R$.

(c) $\left(R_{1} \odot R_{2}\right)^{*}=R_{2}^{*} \odot R_{1}^{*}$.

(d) $R=\left[\begin{array}{ll}D & C \\ B & A\end{array}\right]$ has a 'Grasse inverse' $S$ if and only if $D^{-1}$ exists. It satisfies $R \odot S=S \odot R=E$ and is given by

$$
S=\left[\begin{array}{cc}
D^{-1} & -D^{-1} C \\
-B D^{-1} & -\left(A-B D^{-1} C\right)
\end{array}\right] \text {. }
$$

If $R$ corresponds to $G(s)$ then $S$ corresponds to $G(-s)^{-1}$. (e) $R=R_{1} \odot R_{2}$ has a Grasse inverse $S$ if and only if $R_{1}$ has a Grasse inverse $S_{1}$ and $R_{2}$ has a Grasse inverse $S_{2}$; it satisfies $S=S_{2} \odot S_{1}$.

A $(p+n) \times(p+n)$ realization matrix $R$ will be called Grasse unitary if it holds that $R \odot R^{*}=E$. Note that the set of Grasse unitary matrices forms a group: the Grasse product of two Grasse unitary matrices yields a Grasse unitary matrix. In connection with realization matrices corresponding to (balanced) realizations of (continuoustime) lossless functions, the following properties hold.

Proposition 2. (a) If $R$ corresponds to a (minimal) balanced realization of a lossless transfer function, then $R$ is Grasse unitary.

(b) Conversely, if $R$ is Grasse unitary then $R$ corresponds to a lossless transfer function. The associated realization $(A, B, C, D)$ is balanced (hence minimal) if and only if in addition $A$ is asymptotically stable.

\subsection{LFTs and J-lossless matrices}

Let $\Theta(s)=\left[\begin{array}{cc}\Theta_{1}(s) & \Theta_{2}(s) \\ \Theta_{3}(s) & \Theta_{4}(s)\end{array}\right]$ be a $2 p \times 2 p$ block-partitioned matrix function with blocks of size $p \times p$. Then the linear fractional transformation (LFT) $T_{\Theta}$ associated with $\Theta(s)$ is defined to take any $p \times p$ matrix $G(s)$ for which $\Theta_{3}(s) G(s)+\Theta_{4}(s)$ is invertible to the $p \times p$ matrix given by

$$
T_{\Theta}(G)(s)=\left[\Theta_{1}(s) G(s)+\Theta_{2}(s)\right]\left[\Theta_{3}(s) G(s)+\Theta_{4}(s)\right]^{-1} .
$$

If $G(s)$ is rational of McMillan degree $n$ and $\Theta(s)$ is rational of McMillan degree $m$, then $T_{\Theta}(G)$ is rational of McMillan degree $\leq n+m$. If $\Theta(s)$ and $\Phi(s)$ are two $2 p \times 2 p$ matrix functions, then the LFT $T_{\Theta \Phi}=T_{\Theta} \circ T_{\Phi}$ is a composition of LFTs (on the intersection of domains where both expressions are well defined). In particular: $T_{\Theta}^{-1}=T_{\Theta^{-1}}$.

When dealing with a domain of lossless functions $G(s)$ it is useful to consider $J$-lossless matrices $\Theta(s)$ and their realizations, where $J=\left[\begin{array}{cc}I_{p} & 0 \\ 0 & -I_{p}\end{array}\right]$. Here, a $2 p \times 2 p$ matrix $\Theta(s)$ is called (continuous-time) $J$-lossless if it holds that

$$
\begin{array}{ll}
\Theta(s) J \Theta(s)^{*} \leq J & \text { for all } s \in \Pi^{+}, \\
\Theta(s) J \Theta(s)^{*}=J & \text { for all } s \in i \mathbb{R}, \\
\Theta(s) J \Theta(s)^{*} \geq J & \text { for all } s \in \Pi^{-} .
\end{array}
$$


A $J$-lossless matrix $\Theta(s)$ is invertible; its inverse is given by $\Theta(s)^{-1}=J \Theta^{\sharp}(s) J$. If a $J$-lossless matrix is proper rational, then it has a (minimal) $J$-balanced realization $(\mathcal{A}, \mathcal{B}, \mathcal{C}, \mathcal{D})$, i.e., which satisfies

$$
\begin{aligned}
& \mathcal{D} J \mathcal{D}^{*}=J(\mathcal{D} \text { is constant } J \text {-unitary }), \\
& \mathcal{C}=-\mathcal{D J B}^{*}(\text { of size } 2 p \times m), \\
& \mathcal{A}+\mathcal{A}^{*}=-\mathcal{B J B}^{*} .
\end{aligned}
$$

We now have the following result.

Theorem 3. If $G(s)$ is $p \times p$ lossless of McMillan degree $n$ and the $2 p \times 2 p$ matrix $\Theta(s)$ is $J$-lossless of McMillan degree $m$, then $F(s)=T_{\Theta(s)}(G(s))$ is $p \times p$ lossless of McMillan degree $\leq n+m$.

\section{PARAMETRIZATION OF LOSSLESS MATRICES USING THE TANGENTIAL SCHUR ALGORITHM}

The following theorem describes one iteration step in the continuous-time version of the tangential Schur algorithm, where the purpose is to characterize a lossless function $F(s)$ of McMillan degree $n \geq 1$ satisfying a given interpolation condition, in terms of an LFT on a lossless function $G(s)$ of McMillan degree $n-1$.

Definition 4. Let $w \in \Pi^{+}$be an arbitrary interpolation point, $u \in \mathbb{C}^{p}$ with $\|u\|=1$ a normalized direction vector, and $v \in \mathbb{C}^{p}$ with $\|v\|<1$ a Schur vector. Then the $2 p \times 2 p$ matrix function $\Theta_{w, u, v}(s)$ is defined as

$$
\Theta_{w, u, v}(s)=I_{2 p}+\frac{(w+\bar{w})}{(s-w)} \frac{\left[\begin{array}{l}
v \\
u
\end{array}\right]\left[\begin{array}{l}
v \\
u
\end{array}\right]^{*} J}{\left(\|v\|^{2}-\|u\|^{2}\right)} .
$$

This matrix function is $J$-lossless of McMillan degree 1 having its pole at $w$.

Theorem 5. Let $F(s)$ be a $p \times p$ lossless function of McMillan degree $n \geq 1$. Let $w \in \Pi^{+}$be an arbitrary interpolation point. Then there exists a normalized direction vector $u \in \mathbb{C}^{p}$ with $\|u\|=1$, for which the Schur vector $v:=F(w) u$ satisfies $\|v\|<1$.

For such choices of $w, u$, and $v$, there exists a unique $p \times p$ lossless function $G(s)$ of McMillan degree $n-1$ for which

$$
F=T_{\Theta_{w, u, v}}(G) \text {. }
$$

The tangential Schur algorithm yields, from a lossless function $F(s)$ of McMillan degree $n$, a finite sequence of Schur vectors $\mathbf{v}=\left(v_{n}, v_{n-1}, \ldots, v_{1}\right)$ and a unitary $p \times p$ matrix $F_{0}$, associated with

- a sequence $\mathbf{w}=\left(w_{n}, w_{n-1}, \ldots, w_{1}\right)$ of interpolation points in $\Pi^{+}$,

- a sequence $\mathbf{u}=\left(u_{n}, u_{n-1}, \ldots, u_{1}\right)$ of normalized direction vectors with $\left\|u_{k}\right\|=1(k=1, \ldots, n)$.

It works as follows. Let $F_{n}=F$, then for $k=n, n-1, \ldots, 1$ : put $v_{k}=F_{k}\left(w_{k}\right) u_{k}$.

- If $\left\|v_{k}\right\|<1$, let $F_{k-1}=T_{\Theta_{w_{k}, u_{k}, v_{k}}}^{-1} F_{k}$, which has degree $k-1$.

- If $\left\|v_{k}\right\|=1$ then stop.

A local coordinate map $\varphi(\mathbf{w}, \mathbf{u})$ can be associated with the sequences $\mathbf{w}$ and $\mathbf{u}$ and with $F_{0}$ by

$\varphi(\mathbf{w}, \mathbf{u}): \quad F(s) \in \mathcal{V}(\mathbf{w}, \mathbf{u}) \mapsto\left(v_{1}, v_{2}, \ldots, v_{k}, F_{0}\right)$, where the domain $\mathcal{V}(\mathbf{w}, \mathbf{u})$ of the map consists of functions $F$ for which $\left\|v_{k}\right\|<1$ for all $k=n, n-1 \ldots, 1$ and $F_{0} \in U(n)$.

\subsection{Computing balanced realizations}

In Peeters et al. (2001) a unified framework is presented in which linear fractional transformations on transfer functions are represented by corresponding linear fractional transformation on state-space realization matrices. We have the following result (Peeters et al., 2001, Thm. 8):

Theorem 6 . Let $G(s)$ be a $p \times p$ proper rational matrix function of McMillan degree $n$, and let $(A, B, C, D)$ be a minimal state-space realization of $G$. Let

$$
\Theta=\left[\begin{array}{ll}
\Theta_{11} & \Theta_{12} \\
\Theta_{21} & \Theta_{22}
\end{array}\right]
$$

be a partitioned $2 p \times 2 p$ rational matrix function of McMillan degree $m$ which is proper, and let $(\mathcal{A}, \mathcal{B}, \mathcal{C}, \mathcal{D})$ be a minimal state-space realization of $\Theta$ partitioned into blocks of size $p \times p$ :

$$
(\mathcal{A}, \mathcal{B}, \mathcal{C}, \mathcal{D})=\left(\mathcal{A},\left[\mathcal{B}_{1} \mathcal{B}_{2}\right],\left[\begin{array}{l}
\mathcal{C}_{1} \\
\mathcal{C}_{2}
\end{array}\right],\left[\begin{array}{ll}
\mathcal{D}_{11} & \mathcal{D}_{12} \\
\mathcal{D}_{21} & \mathcal{D}_{22}
\end{array}\right]\right)
$$

Then a state-space realization $(\tilde{A}, \tilde{B}, \tilde{C}, \tilde{D})$ of $F=T_{\Theta}(G)$ is obtained by

$$
\left[\begin{array}{ll}
\tilde{D} & \tilde{C} \\
\tilde{B} & \tilde{A}
\end{array}\right]=T_{\Delta}\left(R_{e}\right), \quad \text { with } R_{e}=\left[\begin{array}{ccc}
D & 0 & C \\
0 & I_{m} & 0 \\
B & 0 & A
\end{array}\right]
$$

and

$$
\left.\Delta=\left[\begin{array}{ccc|ccc}
\mathcal{D}_{11} & \mathcal{C}_{1} & 0 & \mathcal{D}_{12} & 0 & 0 \\
\mathcal{B}_{1} & \mathcal{A} & 0 & \mathcal{B}_{2} & 0 & 0 \\
0 & 0 & I_{n} & 0 & 0 & 0 \\
\hline \mathcal{D}_{21} & \mathcal{C}_{2} & 0 & \mathcal{D}_{22} & 0 & 0 \\
0 & 0 & 0 & 0 & I_{m} & 0 \\
0 & 0 & 0 & 0 & 0 & I_{n}
\end{array}\right]\right]
$$

This theorem directly applies to the situation at hand and takes a simple form which mimics what we have in discretetime (Hanzon et al., 2006, Thm. I.6.4): the Schur recursion can be computed on the level of state-space realizations by a Grasse product.

Theorem 7. A balanced realization $(\tilde{A}, \tilde{B}, \tilde{C}, \tilde{D})$ of $F=$ $T_{\Theta_{w, u, v}}(G)$ is computed from a balanced realization $(A, B, C, D)$ of $G(s)$ by the formula

$$
\left[\begin{array}{cc}
\widetilde{D} & \widetilde{C} \\
\widetilde{B} & \widetilde{A}
\end{array}\right]=\left[\begin{array}{ll}
V & 0 \\
0 & 0
\end{array}\right] \odot\left[\begin{array}{ccc}
D & 0 & C \\
0 & 0 & 0 \\
B & 0 & A
\end{array}\right] \odot\left[\begin{array}{ll}
U & 0 \\
0 & 0
\end{array}\right]^{*},
$$

in which $U$ and $V$ are two $(p+1) \times(p+1)$ Grasse unitary matrices, defined in terms of $w, u$ and $v$ by

$$
U=\left[\begin{array}{cc}
I_{p} & \sqrt{\frac{(w+\bar{w})}{1-\|v\|^{2}}} u \\
-\sqrt{\frac{(w+\bar{w})}{1-\|v\|^{2}}} u^{*} & -\frac{1}{2} \frac{(w+\bar{w})}{\left(1-\|v\|^{2}\right)}
\end{array}\right]
$$

and

$$
V=\left[\begin{array}{cc}
I_{p} & \sqrt{\frac{(w+\bar{w})}{1-\|v\|^{2}}} v \\
-\sqrt{\frac{(w+\bar{w})}{1-\|v\|^{2}}} v^{*}-\frac{1}{2}\left[\frac{\|v\|^{2}(w+\bar{w})}{\left(1-\|v\|^{2}\right)}+\bar{w}-w\right]
\end{array}\right]
$$


Proof. Note that $\frac{(w+\bar{w})}{1-\|v\|^{2}}$ is a positive real number and let $\lambda$ be its positive square root. A $J$-balanced realization of $\Theta_{w, u, v}$ is given by

$$
\mathcal{D}=I_{2 p}, \mathcal{C}=\lambda\left[\begin{array}{l}
v \\
u
\end{array}\right], \mathcal{B}=-\lambda\left[\begin{array}{l}
v \\
u
\end{array}\right]^{*} J, \mathcal{A}=w .
$$

Applying Thm. 6 yields

$$
\left[\begin{array}{cc}
\widetilde{D} & \widetilde{C} \\
\widetilde{B} & \widetilde{A}
\end{array}\right]=\left[\begin{array}{ccc}
D & \lambda(v-D u) & C \\
\lambda\left(u^{*}-v^{*} D\right) & w-\lambda^{2}\left(1-v^{*} D u\right) & -\lambda v^{*} C \\
B & -\lambda B u & A
\end{array}\right]
$$

The same expression is obtained when working out the Grasse product (26) using definitions (27) and (28).

\section{INTERPOLATION OF CONTINUOUS-TIME LOSSLESS FUNCTIONS ON THE IMAGINARY AXIS}

\subsection{Boundary interpolation}

Lemma 8. Let $F(s)$ be a $p \times p$ rational lossless function, $\sigma \in i \mathbb{R}$ a point on the imaginary axis, and $u \in \mathbb{C}^{p}$ a given normalized direction vector. Then the scalar expression

$$
\rho=-u^{*} F(\sigma)^{*} F^{\prime}(\sigma) u,
$$

is nonnegative real: $\rho \geq 0$.

A well-posed boundary interpolation problem not only involves a Schur vector $v$, but it also includes the value $\rho$ of the so-called angular derivative. This is due to the fact that $\|v\|=1$, because $F(\sigma)$ is unitary on the boundary $i \mathbb{R}$, causing one real degree of freedom to occur (now captured by $\rho)$. It can be stated as follows:

Problem 9. (GADI) Characterize all the rational lossless functions $F(s)$ of McMillan degree $n \geq 1$ satisfying the interpolation conditions

$$
F(\sigma) u=v, \quad v^{*} F^{\prime}(\sigma) u=-\rho
$$

where $\sigma \in i \mathbb{R}, u$ and $v$ are unit vectors, and $\rho>0$ is the angular derivative.

Any solution of this generalized angular derivative interpolation problem can be represented as a linear fractional transformation of a lossless function $G(s)$ of McMillan degree $n-1$ such that $G(\sigma) u \neq v$, see Ball et al. (1990).

\subsection{Description of the solutions}

The $J$-lossless matrix function associated with the LFT involved in the solution of the GADI Problem is, up to a constant $J$-unitary right multiplier, given by

$$
\Phi_{\sigma, u, v, \rho}(s)=I_{2 p}-\frac{1}{\rho(s-\sigma)}\left[\begin{array}{l}
v \\
u
\end{array}\right]\left[\begin{array}{l}
v \\
u
\end{array}\right]^{*} J .
$$

Then the following result holds.

Theorem 10. (a) Let $\sigma \in i \mathbb{R}, u \in \mathbb{C}^{p}$ with $\|u\|=1$, $v \in \mathbb{C}^{p}$ with $\|v\|=1$, and $\rho \in \mathbb{R}^{+}$. Let $\Phi_{\sigma, u, v, \rho}$ be the elementary $J$-lossless factor given by $(33)$. Let $G(s)$ be a $p \times p$ rational lossless function of McMillan degree $n$ such that $G(\sigma) u \neq v$. Let $F(s)$ be defined by $F=T_{\Phi_{\sigma, u, v}}(G)$. Then $F(s)$ is a $p \times p$ rational lossless function of McMillan degree $n+1$ such that

$$
F(\sigma) u=v, \quad v^{*} F^{\prime}(\sigma) u=-\rho .
$$

(b) Let $F(s)$ be a $p \times p$ rational lossless function of McMillan degree $n+1$. Let $\sigma \in i \mathbb{R}$, and let $u \in \mathbb{C}^{p}$ with $\|u\|=1$ be such that $\rho:=-u^{*} F(\sigma)^{*} F^{\prime}(\sigma) u$ is strictly positive. Let $v:=F(\sigma) u$ (which satisfies $\|v\|=1$ ), and let $\Phi_{\sigma, u, v, \rho}$ be the elementary $J$-lossless factor (33). Let $G(s)$ be defined by $G=T_{\Phi_{\sigma, u, v}}^{-1}(F)$. Then $G(s)$ is a $p \times p$ rational lossless function of McMillan degree $n$ such that $G(\sigma) u \neq v$.

\subsection{Balanced state-space realizations}

Applying the approach developed in Section 3.1 we have the following result.

Theorem 11. A balanced realization $(\tilde{A}, \tilde{B}, \tilde{C}, \tilde{D})$ of $F=$ $T_{\Phi_{\sigma, u, v, \rho}}(G)$ is computed from a balanced realization $(A, B, C, D)$ of $G(s)$ by the formula

$$
\left[\begin{array}{cc}
\widetilde{D} & \widetilde{C} \\
\widetilde{B} & \widetilde{A}
\end{array}\right]=\left[\begin{array}{ll}
V & 0 \\
0 & 0
\end{array}\right] \odot\left[\begin{array}{ccc}
D & 0 & C \\
0 & 0 & 0 \\
B & 0 & A
\end{array}\right] \odot\left[\begin{array}{ll}
U & 0 \\
0 & 0
\end{array}\right]^{*},
$$

in which $U$ and $V$ are the Grasse unitary matrices defined in terms of $w, u$ and $v$ by

$$
U=\left[\begin{array}{cc}
I_{p} & \frac{1}{\sqrt{\rho}} u \\
-\frac{1}{\sqrt{\rho}} u^{*} & -\frac{1}{2 \rho}
\end{array}\right], \quad V=\left[\begin{array}{cc}
I_{p} & \frac{1}{\sqrt{\rho}} v \\
-\frac{1}{\sqrt{\rho}} v^{*} & \sigma-\frac{1}{2 \rho}
\end{array}\right] .
$$

Note that the recursion step (35) can be rewritten as

$$
\left[\begin{array}{cc|cc}
\widetilde{D} & \widetilde{C} \\
\widetilde{B} & \widetilde{A}
\end{array}\right]=\left[\begin{array}{c|cc}
D & \frac{v-D u}{\sqrt{\rho}} & C \\
\hline \frac{u^{*}-v^{*} D}{\sqrt{\rho}} & \sigma-\frac{1-v^{*} D u}{\rho} & -\frac{v^{*} C}{\sqrt{\rho}} \\
B & -\frac{B u}{\sqrt{\rho}} & A
\end{array}\right],
$$

and that boundary interpolation can be viewed as a limit of classical interpolation by observing that

$$
\lim _{w \rightarrow \sigma} \frac{1-\|F(w) u\|^{2}}{w+\bar{w}}=\rho .
$$

\section{THE SCALAR CASE: BOUNDARY}

\section{INTERPOLATION AND OBER'S CANONICAL FORM}

\subsection{Ober's canonical form}

In the scalar case, a real continuous-time lossless scalar function $G(s)$ of McMillan degree $n$ has a unique realization $(A, b, c, d)$ with $A$ in Schwarz form, see Ober (1987a); see also Ober $(1987 \mathrm{~b}, 1991)$. The realization matrix $R$ is then given by

$$
R=\left[\begin{array}{c|ccccc}
-\epsilon & \epsilon \beta & 0 & \cdots & & 0 \\
\hline \beta & -\frac{\beta^{2}}{2} & \alpha_{1} & & & \\
0 & -\alpha_{1} & 0 & & & \\
\vdots & & & \ddots & & \\
& & & & 0 & \alpha_{n-1} \\
0 & & & & -\alpha_{n-1} & 0
\end{array}\right]
$$

with $\epsilon= \pm 1$, and $\beta, \alpha_{1}, \alpha_{2}, \ldots, \alpha_{n-1}$ all positive real numbers.

Let $A_{n-k}$ be defined as the tridiagonal matrix

$$
A_{n-k}=\left[\begin{array}{ccccc}
0 & \alpha_{k+1} & & 0 \\
-\alpha_{k+1} & 0 & & & \\
& \ddots & \ddots & \ddots & \\
& & & 0 & \alpha_{n-1} \\
0 & & & -\alpha_{n-1} & 0
\end{array}\right]
$$


of which the characteristic polynomial is denoted by $\Delta_{n-k}(s)=\operatorname{det}\left(s I_{n-k}-A_{n-k}\right)$. Then $\Delta_{j}$ is a monic polynomial, which is even (odd) for $j$ even (odd), and it satisfies the 2 nd order recursion

$$
\Delta_{n-k}(s)=s \Delta_{n-k-1}(s)+\alpha_{k+1}^{2} \Delta_{n-k-2}(s) .
$$

It then holds that $G(s)$ equals

$$
G(s)=-\epsilon \frac{\Delta_{n}(s)-\frac{\beta^{2}}{2} \Delta_{n-1}(s)}{\Delta_{n}(s)+\frac{\beta^{2}}{2} \Delta_{n-1}(s)} .
$$

$G(s)$ satisfies interpolation conditions at $\infty$ on the stability boundary:

$$
G(\infty)=-\epsilon, \quad \lim _{s \rightarrow \infty} s^{2} G^{\prime}(s)=\epsilon \beta^{2} .
$$

It can be generated from the Schur algorithm for boundary interpolation at infinity, as explained below; see also Hanzon et al. (2008).

\subsection{Boundary interpolation on the imaginary axis and a Schur algorithm}

The corresponding well-posed angular derivative interpolation (ADI) problem, see Ball et al. (1990), describes the scalar version of the GADI Problem 9. For a scalar lossless function $f(s)$, the conditions (32) reduce to:

$$
f(\sigma)=\xi, \quad f^{\prime}(\sigma)=-\xi \rho
$$

with $\sigma \in i \mathbb{R}$ and $\xi=v / u$ satisfying $|\xi|=1$. The angular derivative $\rho$ satisfies $\rho>0$ if the McMillan degree of $f(s)$ is $\geq 1$. All the solutions are then given by

$$
f=T_{\Theta, \rho, \xi}(g)
$$

where $\Theta_{\sigma, \rho, \xi}(s)$ is the $J$-lossless matrix function

$$
\Theta_{\sigma, \rho, \xi}(s)=J-\frac{1}{\rho(s-\sigma)}\left[\begin{array}{l}
\xi \\
1
\end{array}\right]\left[\begin{array}{l}
\xi \\
1
\end{array}\right]^{*}
$$

with $J=\left[\begin{array}{cc}1 & 0 \\ 0 & -1\end{array}\right]$. Here $g(s)$ ranges over the lossless functions of McMillan degree $\operatorname{deg}(g)=\operatorname{deg}(f)-1$ which satisfy $g(\sigma) \neq-\xi$. (Compared to the expression (33) we have included the right multiplier $J$, which accounts for the changed sign in the condition $g(\sigma) \neq-\xi$.)

A Schur algorithm for real scalar lossless functions with the interpolation points all located at the origin $(\sigma=0)$ is now given by the recursion for $j=n, n-1, \ldots, 1$ :

$$
\begin{aligned}
& f_{j}(0)=\xi, \quad f_{j}^{\prime}(0)=-\xi \rho_{j}, \\
& f_{j-1}=T_{\Theta_{\sigma_{j}, \rho_{j}, \xi}}^{-1}\left(f_{j}\right) .
\end{aligned}
$$

It takes $f=f_{n}$ to the set of parameters $\left(\xi, \rho_{1}, \rho_{2}, \ldots, \rho_{n}\right)$ with $\xi=f(0)= \pm 1$.

\subsection{Connection with Ober's canonical form}

To move the interpolation points from $\sigma=0$ to $\infty$, let $F_{j}(s)=f_{j}(1 / s)$ (which is also lossless) and compute $F(s)=F_{n}(s)$ using the reversed Schur algorithm from $F_{0}=\xi=1$. Let $a_{j}=\rho_{j} / 2$. Separating the odd and even parts of the numerators and the denominators, we get $F_{j}(s)=\frac{P_{j}-a_{j} P_{j-1}}{P_{j}+a_{j} P_{j-1}}$, where $P_{j}$ is a monic polynomial, which is even (odd) for $j$ even (odd), satisfying the 2nd order recursion

$$
P_{j+1}(s)=s P_{j}(s)+a_{j} a_{j+1} P_{j-1}(s) .
$$

Comparing with Eqn. (41) it follows that

$$
\beta^{2}=\rho_{n}, \quad \alpha_{n-j}^{2}=a_{j} a_{j+1} .
$$

For $j=1, \ldots, n$ a balanced realization of $F_{j}(s)$ is obtained by the following recursion from Thm. 11:

$$
\left[\begin{array}{ll}
d_{j} & c_{j} \\
b_{j} & A_{j}
\end{array}\right]=\left[\begin{array}{c|cc}
1 & -\sqrt{\rho_{j}} & 0 \\
\hline \sqrt{\rho_{j}} & -\rho_{j} / 2 & \frac{\sqrt{\rho_{j}}}{2} c_{j-1} \\
0 & \frac{\sqrt{\rho_{j}}}{2} b_{j-1} & A_{j-1}-\frac{b_{j-1} c_{j-1}}{2}
\end{array}\right]
$$

which recovers (up to a change of signs in $A_{n}$ ) the balanced canonical form of Ober, with $\epsilon=-1$.

\section{THE MULTIVARIABLE CASE: INTERPOLATION AT INFINITY AND PIVOT STRUCTURES}

In the multivariable case we can also map $s$ to $1 / s$, to move the interpolation condition from $\sigma=0$ to $\infty$.

Theorem 12. Let $F(s)$ be a $p \times p$ rational lossless function of McMillan degree $n+1$. Let $\sigma \in i \mathbb{R}$. Choose $u \in \mathbb{C}^{p}$ with $\|u\|=1$ such that $\rho:=\lim _{s \rightarrow \infty} s^{2} u^{*} F(s)^{*} F^{\prime}(s) u$ is strictly positive. Define $v:=F(\infty) u$ (which satisfies $\|v\|=1$ ), and let

$$
\Psi_{u, v, \rho}(s)=I_{2 p}-\frac{s}{\rho}\left[\begin{array}{l}
v \\
u
\end{array}\right]\left[\begin{array}{l}
v \\
u
\end{array}\right]^{*} J .
$$

Then $F=T_{\Psi_{u, v, \rho}}(G)$ for some $p \times p$ rational lossless function $G(s)$ of McMillan degree $n$ such that $G(\infty) u \neq v$.

Now, the $J$-lossless function $\Psi_{u, v, \rho}(s)$ fails to be proper, but $\Psi_{u, v, \rho}(1 / s)=\Phi_{0, u, v, \rho}(s)$ is proper. Thm. 8 of Peeters et al. (2001) also allows one to deal with this situation and to compute a balanced realization $(\tilde{A}, \tilde{B}, \tilde{C}, \tilde{D})$ of $F(s)$ from a balanced realization $(A, B, C, D)$ of $G(s)$ using a $J$-balanced realization of $\Phi_{0, u, v, \rho}(s)$ :

Theorem 13. A balanced realization $(\tilde{A}, \tilde{B}, \tilde{C}, \tilde{D})$ of $F=$ $T_{\Phi_{0, u, v, \rho}}(G)$ is computed from a balanced realization $(A, B, C, D)$ of $G(s)$ by

$$
\left[\begin{array}{cc}
\widetilde{D} & \widetilde{C} \\
\widetilde{B} & \widetilde{A}
\end{array}\right]=\left[\begin{array}{ccc}
D & 0 & C \\
0 & 0 & 0 \\
B & 0 & A
\end{array}\right]-\frac{1}{1-v^{*} D u}\left[\begin{array}{c}
D u-v \\
-\sqrt{\rho} \\
B u
\end{array}\right]\left[\begin{array}{c}
u-D^{*} v \\
-\sqrt{\rho} \\
-C^{*} v
\end{array}\right]^{*}
$$

Proof. By a direct application of (Peeters et al., 2001, Thm. 8) we get that the realization matrix of $T_{\Theta}(G)$ is given by

$$
\left[\begin{array}{ccc}
D-\frac{(D u-v)\left(u^{*}-v^{*} D\right)}{1-v^{*} D u} & \frac{\sqrt{\rho}(D u-v)}{1-v^{*} D u} & C+\frac{(D u-v) v^{*} C}{1-v^{*} D u} \\
\frac{\sqrt{\rho}\left(u^{*}-v^{*} D\right)}{1-v^{*} D u} & -\frac{\rho}{1-v^{*} D u} & -\frac{\sqrt{\rho} v^{*} C}{1-v^{*} D u} \\
B-\frac{B u\left(u^{*}-v^{*} D\right)}{1-v^{*} D u} & \frac{\sqrt{\rho} B u}{1-v^{*} D u} & A+\frac{B u v^{*} C}{1-v^{*} D u}
\end{array}\right] .
$$

Note that $\widetilde{D} u=v$ and $\widetilde{B} u=\left[\begin{array}{c}\sqrt{\rho} \\ 0\end{array}\right]$, whence $u^{*} \widetilde{B}^{*} \widetilde{B} u=\rho$. If $u$ is chosen to be a standard basis vector $e_{k}$, then the $k$ th column of $\widetilde{B}$ is a pivot- 1 column. However, if $[B, A]$ has a pivot structure, then $[\widetilde{B}, \widetilde{A}]$ will only have a pivot structure for every choice of $v$ if $B u$ is a pivot-1 column too. This confirms that pivot structures are indeed obtained in the scalar case (when $k=1$ is the only option). But in the multivariable case a pivot structure is only generated in this way when the same choice $u=e_{k}$ is 
made in every iteration of the (reversed) tangential Schur algorithm. (Then only column $k$ of $B$ (and $\widetilde{B}$ ) will be a pivot column, while $A$ (and $\widetilde{A}$ ) is upper-Hessenberg.) This does not admit the generation of an atlas of pivot structures.

To construct an atlas of pivot structures for input-normal pairs $(A, B)$, the following modifications to the above procedure can be made. (1) We address the quotient group of lossless systems for which $D=I_{p}$, with balanced realizations of the form $\left(A, B,-B^{*}, I_{p}\right)$. (2) The specific choice $v=-u$ is made, which implies $D u-v=2 u$, $u^{*}-v^{*} D=2 u^{*}$ and $1-v^{*} D u=2$. This initially gives $\widetilde{D}=I_{p}-2 u u^{*}$. (3) An additional LFT with a constant $J$ unitary multiplier $H$ is applied to re-obtain $\widetilde{D}=I_{p}$ and to generate (and parameterize) a pivot structure for $[B, A]$.

Theorem 14. Let $H$ be the constant $J$-unitary matrix

$$
H=\left[\begin{array}{cc}
I_{p}-2 u u^{*}-h u^{*}-u h^{*}+\mu u u^{*} & -h u^{*}+\mu u u^{*} \\
-u h^{*}+\mu u u^{*} & I_{p}+\mu u u^{*}
\end{array}\right]
$$

with $h$ such that $h^{*} u=0, \mu=\frac{1}{2} h^{*} h+\gamma$, and $\gamma \in i \mathbb{R}$. Then the realization matrix of a balanced realization $\left(\tilde{A}, \tilde{B},-\tilde{B}^{*}, I_{p}\right)$ of $F(s)=T_{H \Phi_{0, u,-u, \rho}(s)}(G(s))$ is computed from a balanced realization $\left(A, B,-B^{*}, I_{p}\right)$ of $G(s)$ as:

$$
\left[\begin{array}{c|cc}
I_{p} & -\sqrt{\rho}(u+h) & -\left(I_{p}-u u^{*}\right) B^{*} \\
\hline \sqrt{\rho}\left(u^{*}+h^{*}\right) & -\frac{1}{2} \rho\left(1+h^{*} h+2 \gamma\right) & -\frac{1}{2} \sqrt{\rho}\left(u^{*}+2 h^{*}\right) B^{*} \\
B\left(I_{p}-u u^{*}\right) & \frac{1}{2} \sqrt{\rho} B u & A+\frac{1}{2} B u u^{*} B^{*}
\end{array}\right]
$$

If in addition $[B, A]$ has an admissible pivot structure (i.e., with $A$ having a staircase structure) and $u=e_{k}$ is an admissible choice for the direction vector, then $[\widetilde{B}, \widetilde{A}]$ again has an admissible pivot structure.

The vectors $h$ and the scalars $\gamma$ of the iteration steps together serve to parameterize all possible admissible pivot structures for $[B, A]$, and to generate them by applying a sequence of LFTs. But to revert the procedure it is not possible to apply a sequence of LFTs, because the associated matrices happen to be singular. Instead, one uses the explicit formula given in the theorem.

Note that an admissible pivot structure for $[B, A]$ implies the controllability matrix to have a pivot structure too. If one drops the admissibility condition, then pivot structures in $[B, A]$ in general are destroyed - which is different from the discrete-time case where subdiagonal pivot structures are obtained.

\section{CONCLUSIONS}

We have presented a tangential Schur algorithm with boundary interpolation and generalized angular derivative conditions for multivariable lossless systems in continuoustime. It is established that it can be regarded as a limit of classical interpolation with interpolation points tending to the imaginary axis. Balanced realizations in terms of the parameters accompany the algorithm. The procedure underlying Ober's canonical form (with interpolation at infinity and triangular pivot structures) is generalized to the multivariable case. It is found that no complete atlas of pivot structures is obtained. However, for input normal pairs an atlas of admissible pivot structures can still be generated with LFTs in a closely related way.

\section{REFERENCES}

Alpay, D., Baratchart, L., and Gombani, A. (1994). On the differential structure of matrix-valued rational inner functions. Op. Th.: Adv. and Appl., 73, 30-66.

Ball, J., Gohberg, I., and Rodman, L. (1990). Interpolation of rational matrix functions, volume 45 of Op. Th.: Adv. and Appl. Birkhäuser.

Bruls, J., Chou, C., Haverkamp, B., and Verhaegen, M. (1999). Linear and non-linear system identification using separable least-squares. Europ. J. Contr., 5(1), 116-128.

Fulcheri, P. and Olivi, M. (1998). Matrix rational $H^{2}$ approximation: A gradient algorithm based on Schur analysis. SIAM J. Contr. Opt., 36(6), 2103-2127.

Hanzon, B. and Ober, R. (1998). Overlapping blockbalanced canonical forms for various classes of linear systems. Lin. Alg. Appl., 281, 171-225.

Hanzon, B., Olivi, M., and Peeters, R. (2006). Balanced realizations of discrete-time stable all-pass systems and the tangential Schur algorithm. Lin. Alg. Appl., 418, 793-820.

Hanzon, B., Olivi, M., and Peeters, R. (2008). Lossless scalar functions: Boundary interpolation, Schur algorithm and Ober's canonical form. In Proc. 47th CDC, Cancun, 9-11 Dec. 2008, 1845-1850.

Hanzon, B., Olivi, M., and Peeters, R. (2009). Subdiagonal pivot structures and associated canonical forms under state isometries. In Proc. 15th IFAC SYSID 2009, SaintMalo, 6-8 July 2009, 1620-1625.

Hanzon, B. and Peeters, R. (2000). Balanced parametrizations of stable SISO all-pass systems in discrete-time. Math. Contr., Sign., and Syst., 13, 240-276.

Leblond, J. and Olivi, M. (1998). Weighted $H^{2}$ approximation of transfer functions. Math. Contr., Sign., and Syst., 11, 28-39.

Ober, R. (1987a). Asymptotically stable allpass transfer functions: canonical form, parametrization and realization. In Proc. IFAC World Congress, Munich.

Ober, R. (1987b). Balanced realizations: canonical form, parametrization, model reduction. Int. J. Contr., 46, 643-670.

Ober, R. (1991). Balanced parametrization of classes of linear systems. SIAM J. Contr. Opt., 29, 1251-1287.

Peeters, R., Hanzon, B., and Olivi, M. (2001). Linear fractional transformations and balanced realization of discrete-time stable all-pass systems. In Proc. 1st IFAC SSSC, Prague, 27-29 Aug. 2001.

Peeters, R., Hanzon, B., and Olivi, M. (2007). Canonical lossless state-space systems: Staircase forms and the Schur algorithm. Lin. Alg. Appl., 425, 404-433.

Peeters, R., Olivi, M., and Hanzon, B. (2009). Balanced realization of lossless systems: Schur parameters, canonical forms and applications. In Proc. 15th IFAC SYSID 2009, Saint-Malo, 6-8 July 2009, 273-283.

Peeters, R., Olivi, M., and Hanzon, B. (2010). Parametrization of matrix-valued lossless functions based on boundary interpolation. In Proc. 19th MTNS 2010, Budapest, 5-9 July 2010, 563-570.

Strang, G. and Nguyen, T. (1996). Wavelets from Filter Banks. Cambridge-Wellesley.

Vaidyanathan, P. and Doğanata, Z. (1989). The role of lossless systems in modern digital signal processing: a tutorial. IEEE Trans. Educ., 32(3), 181-197. 